\title{
Silicon pore optics mirror module production and testing
}

Collon, Maximilien J.; Vacanti, Giuseppe; Barriere, Nicolas; Landgraf, Boris; Guenther, Ramses; Vervest, Mark; Van Der Hoeven, Roy; Chatbi, Abdel; Girou, David; Sforzini, Jessica

Total number of authors:

37

Published in:

Space Telescopes and Instrumentation 2018: Ultraviolet to Gamma Ray

Link to article, DOI:

$10.1117 / 12.2314479$

Publication date:

2018

Document Version

Publisher's PDF, also known as Version of record

Link back to DTU Orbit

Citation (APA):

Collon, M. J., Vacanti, G., Barriere, N., Landgraf, B., Guenther, R., Vervest, M., Van Der Hoeven, R., Chatbi, A., Girou, D., Sforzini, J., Beijersbergen, M. W., Bavdaz, M., Wille, E., Fransen, S., Shortt, B., Ferreira, I., Haneveld, J., Booysen, K., Koelewijn, A., ... Vernani, D. (2018). Silicon pore optics mirror module production and testing. In J-W. A. den Herder, S. Nikzad, \& K. Nakazawa (Eds.), Space Telescopes and Instrumentation 2018: Ultraviolet to Gamma Ray (Vol. 10699). [106990Y] SPIE - International Society for Optical Engineering.

https://doi.org/10.1117/12.2314479

\section{General rights}

Copyright and moral rights for the publications made accessible in the public portal are retained by the authors and/or other copyright owners and it is a condition of accessing publications that users recognise and abide by the legal requirements associated with these rights.

- Users may download and print one copy of any publication from the public portal for the purpose of private study or research.

- You may not further distribute the material or use it for any profit-making activity or commercial gain

- You may freely distribute the URL identifying the publication in the public portal 


\section{Silicon pore optics mirror module production and testing}

\section{Maximilien J. Collon, Giuseppe Vacanti, Nicolas Barriere, Boris Landgraf, Ramses Guenther, et al.}

Maximilien J. Collon, Giuseppe Vacanti, Nicolas Barriere, Boris Landgraf, Ramses Guenther, Mark Vervest, Roy van der Hoeven, Abdel Chatbi, David Girou, Jessica Sforzini, Marco W. Beijersbergen, Marcos Bavdaz, Eric Wille, Sebastiaan Fransen, Brian Shortt, Jeroen Haneveld, Karin Booysen, Arenda Koelewijn, Maurice Wijnperlé, Coen van Baren, Alexander Eigenraam, Peter Müller, Michael Krumrey, Vadim Burwitz, Daniele Spiga, Giovanni Pareschi, Sonny Massahi, Finn Christensen, Desiree Della Monica Ferreira, Giuseppe Valsecchi, Paul Oliver, Ian Chequer, Kevin Ball, Karl-Heinz Zuknik, "Silicon pore optics mirror module production and testing," Proc. SPIE 10699, Space Telescopes and Instrumentation 2018: Ultraviolet to Gamma Ray, 106990Y (18 July 2018); doi: 10.1117/12.2314479

Event: SPIE Astronomical Telescopes + Instrumentation, 2018, Austin, Texas, United States 


\title{
Silicon Pore Optics Mirror Module Production and Testing
}

Maximilien J. Collon ${ }^{*}$, Giuseppe Vacanti ${ }^{1}$, Nicolas M. Barrière ${ }^{1}$, Boris Landgraf ${ }^{1}$, Ramses Günther ${ }^{1}$, Mark Vervest ${ }^{1}$, Luc Voruz ${ }^{1}$, Sjoerd Verhoex ${ }^{1}$, Ljubiša Babić ${ }^{1}$, Roy van der Hoeven ${ }^{1}$, Kim van Straeten, Abdel Chatbi ${ }^{1}$, David Girou ${ }^{1}$, Marco W. Beijersbergen ${ }^{1}$, Marcos Bavdaz ${ }^{2}$, Eric Wille ${ }^{2}$, Sebastiaan Fransen ${ }^{2}$, Brian Shortt ${ }^{2}$, Ivo Ferreira ${ }^{2}$, Jeroen Haneveld ${ }^{3}$, Arenda Koelewijn ${ }^{3}$, Karin Booysen $^{3}$, Maurice Wijnperle ${ }^{3}$, Jan-Joost Lankwarden ${ }^{3}$, Coen van Baren ${ }^{4}$, Alexander Eigenraam ${ }^{4}$, Jan Willem den Herder ${ }^{4}$, Peter Müller ${ }^{5}$, Michael Krumrey ${ }^{5}$, Vadim Burwitz ${ }^{6}$, Giovanni Pareschi ${ }^{7}$, Sonny Massahi ${ }^{8}$, Desirée Della Monica Ferreira ${ }^{8}$, Finn E. Christensen ${ }^{8}$, Giuseppe Valsecchi ${ }^{9}$, Paul Oliver $^{10}$, Ian Chequer ${ }^{10}$, Kevin Ball ${ }^{10}$, Karl-Heinz Zuknik ${ }^{11}$, Dervis Vernani ${ }^{12}$

${ }^{1}$ cosine, Oosteinde 36, 2361 HE Warmond, The Netherlands

${ }^{2}$ European Space Agency, ESTEC, Keplerlaan 1, PO Box 299, 2200 AG Noordwijk, The Netherlands

${ }^{3}$ Micronit Microfluidics B.V., Colosseum 15, 7521 PV Enschede, The Netherlands

${ }^{4}$ SRON, Sorbonnelaan 2, 3584 CA Utrecht, The Netherlands

${ }^{5}$ Physikalisch-Technische Bundesanstalt (PTB), Abbestr. 2-12, 10587 Berlin, Germany

${ }^{6}$ MPI f. extraterrestrische Physik, Giessenbachstrasse 1, 85748 Garching, Germany

${ }^{7}$ INAF Osservatorio Astronomico di Brera, Via E. Bianchi 46 I- 23807, Merate (Lc), Italy

${ }^{8}$ DTU Space, Technical University of Denmark, Building 327, DK - 2800 Kgs. Lyngby, Denmark

${ }^{9}$ Media Lario S.r.l., Località Pascolo, 23842 Bosisio Parini(LC), Italy

${ }^{10}$ Teledyne e2v (UK) Ltd., 106 Waterhouse Lane, Chelmsford, Essex CM1 2QU, England

${ }^{11}$ OHB System AG, Manfred-Fuchs-Straße 1, 82234 Weßling, Germany

${ }^{12}$ Thales Alenia Space Switzerland Ltd, Schaffhauserstrasse 580, 8052 Zürich, Switzerland

\begin{abstract}
Silicon Pore Optics (SPO) has been established as a new type of x-ray optics that enables future x-ray observatories such as Athena. SPO is being developed at cosine with the European Space Agency (ESA) and academic and industrial partners. The optics modules are lightweight, yet stiff, high-resolution X-ray optics, that shall allow missions to reach an unprecedentedly large effective area of several square meters, operating in the 0.2 to $12 \mathrm{keV}$ band with an angular resolution better than 5 arc seconds. In this paper we are going to discuss the latest generation production facilities and we are going to present results of the production of mirror modules for a focal length of $12 \mathrm{~m}$, including $\mathrm{x}$-ray test results.
\end{abstract}

Keywords: X-ray optics, X-ray astronomy, silicon, wafer, stack, pore optics, X-ray telescopes, ATHENA, SPO

\section{INTRODUCTION}

It has been almost 20 years since the launch of the two largest x-ray observatories flown to this day. The European Space Agency (ESA) launched XMM-Newton in December 1999 [1], after NASA had launched Chandra earlier in July [2]. Both observatories are still in operation but are way beyond their originally planned lifetime of 5 years. A successor telescope has been under discussion by the scientific community and the space agencies ever since the year 2000, due to the extraordinary scientific success that both x-ray observatories have achieved, complementing the work of other ground and space based telescopes in an energy band that is only accessible outside the atmosphere of earth. After several iterations (XEUS, IXO) ESA has selected in 2014 the mission Athena [3-6], currently planned to be launched in the early 2030s, as part of its Cosmic Vision program, on the scientific topic of "Hot and Energetic Universe",

*m.collon@ cosine.nl

Space Telescopes and Instrumentation 2018: Ultraviolet to Gamma Ray, edited by Jan-Willem A. den Herder, Shouleh Nikzad, Kazuhiro Nakazawa, Proc. of SPIE Vol. 10699, 106990Y · (c) 2018 SPIE CCC code: $0277-786 X / 18 / \$ 18 \cdot$ doi: $10.1117 / 12.2314479$ 
addressing two key astrophysics questions: How does ordinary matter assemble into the large-scale structures we see today? And how do black holes grow and shape the universe?

To address these questions, Athena requires two newly developed detectors, one imager with spectroscopic capability over a large field of view (WFI [7]), and a second detector providing spatially resolved high-resolution x-ray spectroscopy over a smaller field of view (XIFU [8]). Both detectors, mounted side-by-side on the telescope, require a large ( $3 \mathrm{~m}$ diameter) lens with a focal length of $12 \mathrm{~m}$, imaging $\mathrm{x}$-ray sources in the energy range of 0.2 to $12 \mathrm{keV}$ with high angular resolution (5" half-energy width (HEW) @ $1 \mathrm{keV}$ ), providing an effective area of about $1.5 \mathrm{~m}^{2}$ at $1 \mathrm{keV}$. Traditionally, double-reflection grazing incidence mirrors are used to make an x-ray lens for imaging telescopes [9], consisting of curved and co-aligned super-polished surfaces which are coated to enhance the reflectivity under grazing incidence angles. For Athena this results in a lens design [10] with several hundred square meters of mirror surface with a roughness of less than $5 \AA$, aligned and mounted to focus at $12 \mathrm{~m}$ distance the on-axis light collected by the lens into a circle of $\sim 0.3 \mathrm{~mm}$ diameter. Gravity, the launcher (Ariane 6) and the spacecraft design limit the optics mass to several hundred $\mathrm{kg}$, which means that the mirrors must be very thin $(\sim 170 \mu \mathrm{m})$ and densely packed $(\sim 0.8 \mathrm{~mm}$ radial separation), mounted stiff enough to survive the launch and operation in space. And finally, the production of such an optic must be very cost efficient to fit into the cost cap of Athena and the assembly must be quick enough to meet the timeline for a launch in 2030 [11].

The technology to construct such a lens did not exist in the year 2000, when the first concepts for Athena were developed. Therefore ESA started technology development, resulting in what is now known as Silicon Pore Optics (SPO) $[12,13]$, a novel kind of x-ray optics that makes missions such as Athena or the NASA midex candidate ARCUS [14] possible. It uses commercially available super-polished 12" silicon wafers as basis for making the x-ray mirrors, has a heavily automated assembly process to keep the production of $100.000-150.000$ mirrors cost efficient and it benefits from the large investments of the semiconductor industry into equipment and processes [15].

SPO has demonstrated, over the course of its evolution and following the development of the precursor designs, on representative samples that it has the potential to meet all of the demanding requirements of Athena [16]. Mirror plates were produced, coated, stacked with robots and were assembled into mirror modules. The modules were environmentally tested, measured in x-rays and integrated into a petal structure. With the selection in 2014 of the mission as L2 launch slot, ESA's efforts have intensified to ready SPO for the Mission Adoption Review (MAR) of Athena, scheduled for 2021. A Qualification Model (QM) of the optics, consisting of about 25 mirror modules at three representative radii mounted in a segment of the final optics, has then to demonstrate that the technology can meet not only the performance requirements, but that it can survive launch. Demonstration of this so-called Technology Readiness Level (TRL) of 6 is required by ESA to adopt a mission, and its achievements are evaluated by experts that are outside of the development teams. Given that mass production is required to build the mirror of Athena, an additional requirement to demonstrate a TRL of 6 means that one must demonstrate with the QM that the optics can be produced to cost and to schedule.

To demonstrate such mass production capability takes time, as it requires significant investment into equipment, operators, procedures and training, and it must therefore be done long ahead of the 2021 MAR. In parallel to the mass production preparation, the SPO technology itself has to be further matured, to increase on all of the three representative radii ( $r=1500 \mathrm{~mm}$ "outer", $r=740 \mathrm{~mm}$ "middle" and $r=250 \mathrm{~mm}$ "inner") the mirror area that meets the angular resolution requirement. To achieve mass production capability and an improved process on time for the MAR, ESA has developed a Technology Development Plan (TDP) [17] that is being carried out by European industry and academia. Several activities in this TDP plan concern SPO mirror module development and this paper reports on the progress made in the last year on all of the areas that effect the manufacturing and testing of the SPO mirror modules. We will also discuss the plan to advance the optics further. To highlight the dual aspect of the development program, we split in each section the topic of mass production and of process development.

\section{MIRROR PLATE PRODUCTION}

\subsection{Preparation of plate mass production}

An important aspect of SPO is that the individual curvature of the plate, as required to form a concentric optic, is generated during the stacking process as described later in this paper, which means per ring of Athena (15-20 rings) only two types of mirror plates are required, which differ in length but are otherwise identical. All other plate parameters can be kept the same, greatly facilitating their production process, the logistics and reducing the cost. SPO uses a 
semiconductor mass production process to generate rectangular silicon plates with a wedged silicon oxide layer. We currently produce for the three development radii three types of geometries of plates (see Figure 1), where fully automated ribbing and dicing machines are used to machine grooves into oxidized 12" wafers, leaving ribs and a thin membrane. Etching is performed to remove micro-cracks in the surfaces. The outside of the membrane is the $\mathrm{x}$-ray mirror and on the inside the ribs are used as interconnects to bond during stacking to the next mirror. As the ribs are an integral part of the former wafer, the spacing of the mirrors is fixed to the original wafer thickness of $775 \mu \mathrm{m}$. We have now demonstrated a production capacity of several thousand plates per year and we can control the dimensions of the plates through programming the dicing machines. The mirror thickness used for development is 150 to $170 \mu \mathrm{m}$, the rib width $170 \mu \mathrm{m}$ and the rib pitch is 1 to $2.3 \mathrm{~mm}$. The wider rib pitch is especially important to increase the field of view of the telescope as for example required by the WFI detector: this was demonstrated first on the inner radii plates. We plan for the near future to increase the rib spacing also on the middle and outer radii, after having first improved the performance of the stacks, as discussed later in this paper.
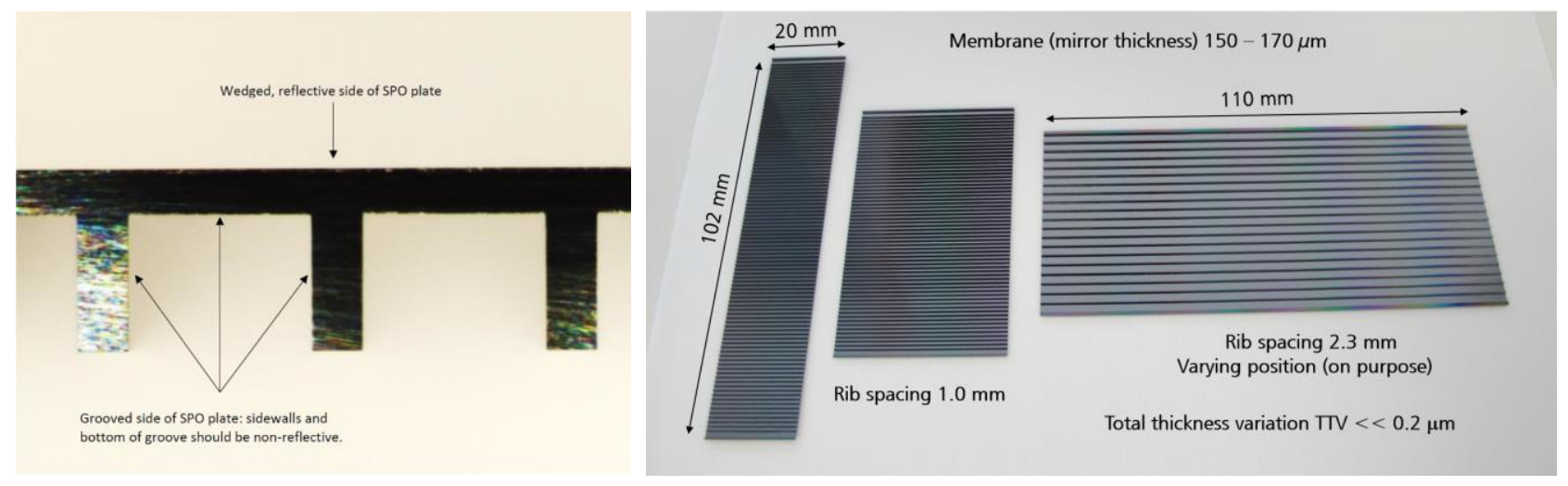

Figure 1 - Cross section of a silicon pore optic mirror plate, showing the ribs and the membrane (Left). The right image shows the three types of plate used for the process development at the outermost radius $(r=1500 \mathrm{~mm}$, left), the middle radius $(r=737 \mathrm{~mm}$, middle) and the innermost radius $(r=250 \mathrm{~mm}$, right). Each plate type demonstrates different aspects of the technological parameter space, as required to pass the Athena MAR. The outer plates are very wide with a $1 \mathrm{~mm}$ wide rib spacing. The innermost radius plates are very long and demonstrate the ability to increase the rib pitch to $2.3 \mathrm{~mm}$ and also the capability to vary the rib position within a plate, such that outer ribs align when plates are bent and stacked. Note that these plate types are technology demonstrators and do not represent the final Athena geometry.

After having increased the mirror plate production capacity at the first plate supplier, in the past year we were able to add a second source of mirror plates. This is important for programmatic reasons, in view of the flight model production. The second supplier has recently produced the first batch of middle radius plates that have been successfully stack (see Figure 2).

\subsection{Plate process development}

We use as starting material for the mirror plate highest grade 12" wafers with a residual total thickness variation of $<$ $200 \mathrm{~nm}$ over the $300 \mathrm{~mm}$ diameter surface, measured by the wafer suppliers, being on the order of $20 \mathrm{~nm}$ on the area of a plate. During the plate production process we alter the surface by growing a silicon oxide of $\sim 2 \mu \mathrm{m}$ into both sides of the wafer, which we wedge [18] down to several hundred $\mathrm{nm}$ with a relative accuracy better than $3 \%$, to be able to later stack plates into a focusing geometry. Optical thin film metrology can accurately measure the oxide layer, and the uncoated wedged plate quality has been determined to be of sufficient quality to meet the requirements of Athena. To advance the plate quality, especially the wedging process, further and to increase the margins in the error budget, we require a system that allows measuring the combined thickness variation of the silicon oxide and the silicon in one go, which is not trivial. We are currently commissioning a system that combines visible and infrared light to measure the absolute thickness variation, which will become operational in the second half of 2018. This system will then be used in the coming year to guide the plate suppliers in the improvement of the wedging process. 

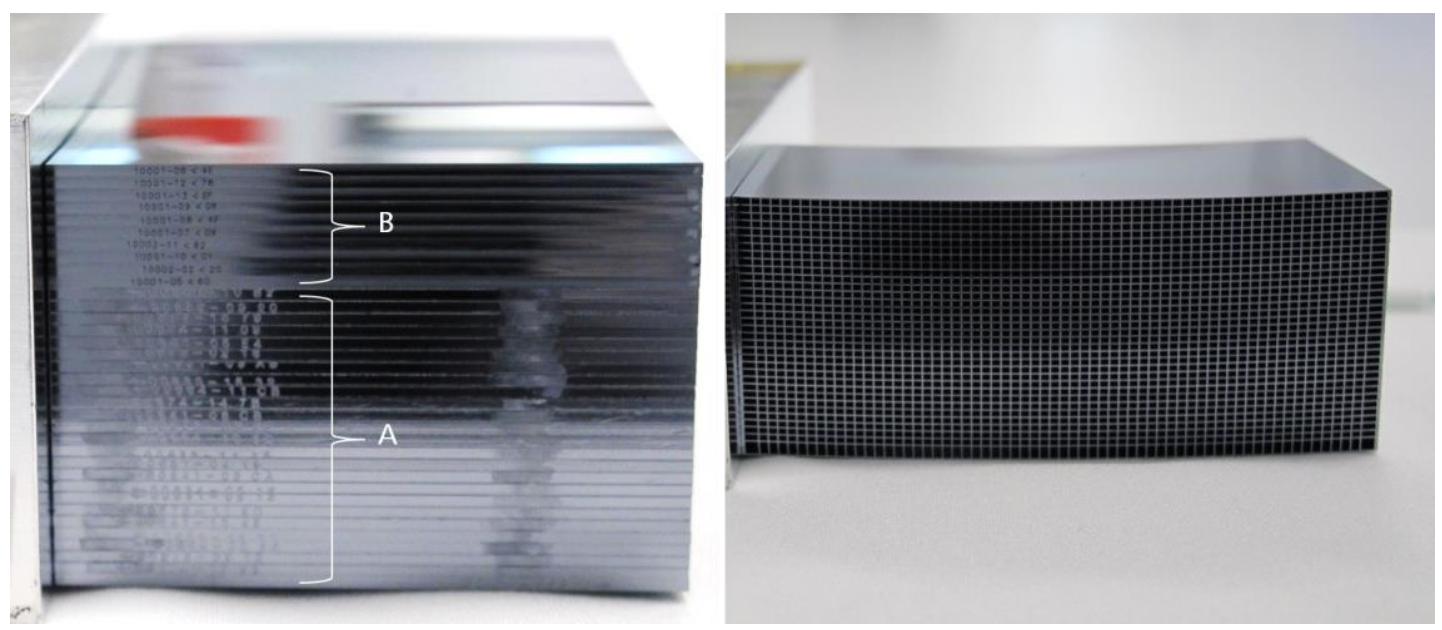

Figure 2 - Photographs of the very first stacks where plates of two different suppliers (A, B) have been stacked on top of each other, demonstrating their compatibility. The left image shows the stack from the side with the different laser marked plate identifier highlighting the two suppliers. The right image shows the front, where no visible difference can been seen.

With the support of SRON and ESA we are also testing different plate process improvements that can benefit Athena in terms of angular resolution, effective area and straylight reduction. One example is the roughening of the ribbed side of the plates, such that the back of the mirror and the side of the ribs have a significantly reduced reflectivity for $\mathrm{x}$-rays, reducing straylight. Figure 3 (left) shows a microscope image of the ribbed side of a test plate, directly after ribbing. We then apply a chemical process which roughens the backside of the mirror and the side of the ribs. In optical wavelengths the silicon appears now darker, as light is absorbed in the etch pits (Figure 3 right). We also tested in x-rays, using the Four-Crystal-Monochromator (FCM) beamline of the Physikalisch-Technische Bundesanstalt (PTB) at the BESSY II synchrotron radiation facility in Berlin, Germany [19]. We performed reflectivity measurements in $\theta / 2 \theta$ configuration on the side of the ribs and the backside of the mirror (membrane) at $2.5 \mathrm{keV}$ and at $10 \mathrm{keV}$. The results (see Figure 4), comparing the reflectivity of a regular plate to the roughened one, show a reduction of reflectivity by several orders of magnitude, for both measured energies.
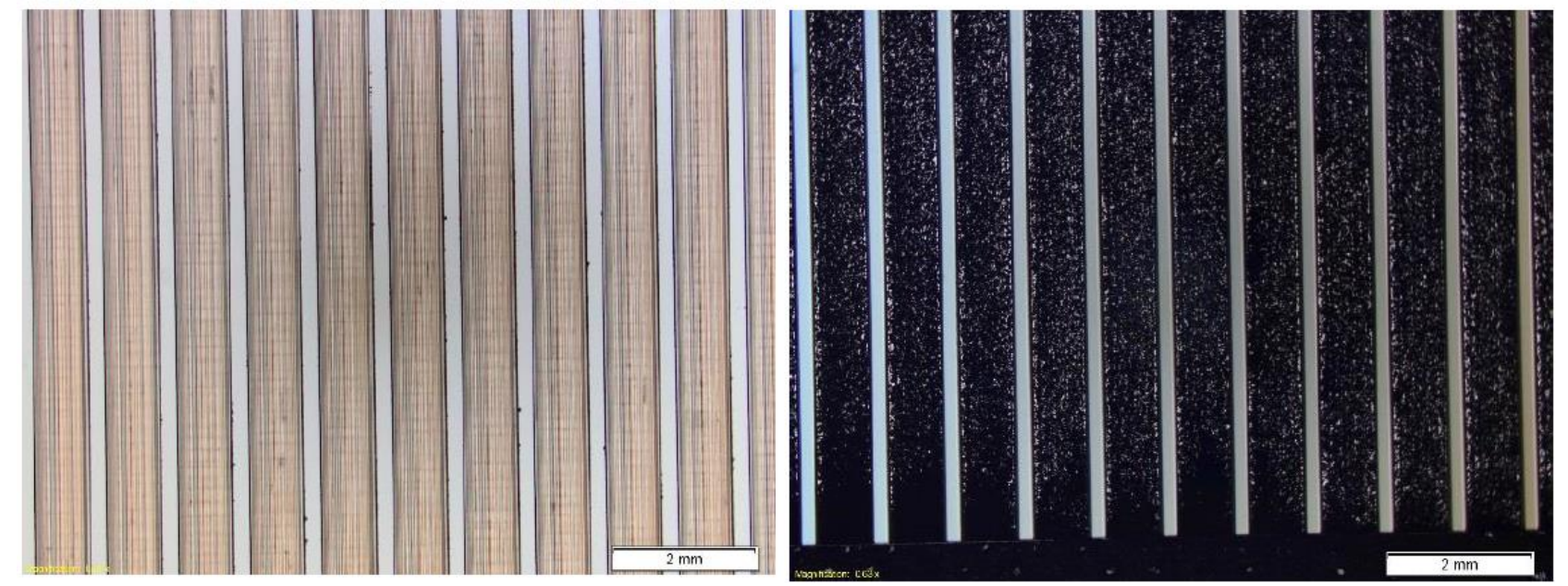

Figure 3 - Microscope images of a middle radius plate, showing the ribbed side. The left image shows the plate before roughening of the non-reflective side. The right image shows the plate after processing. 

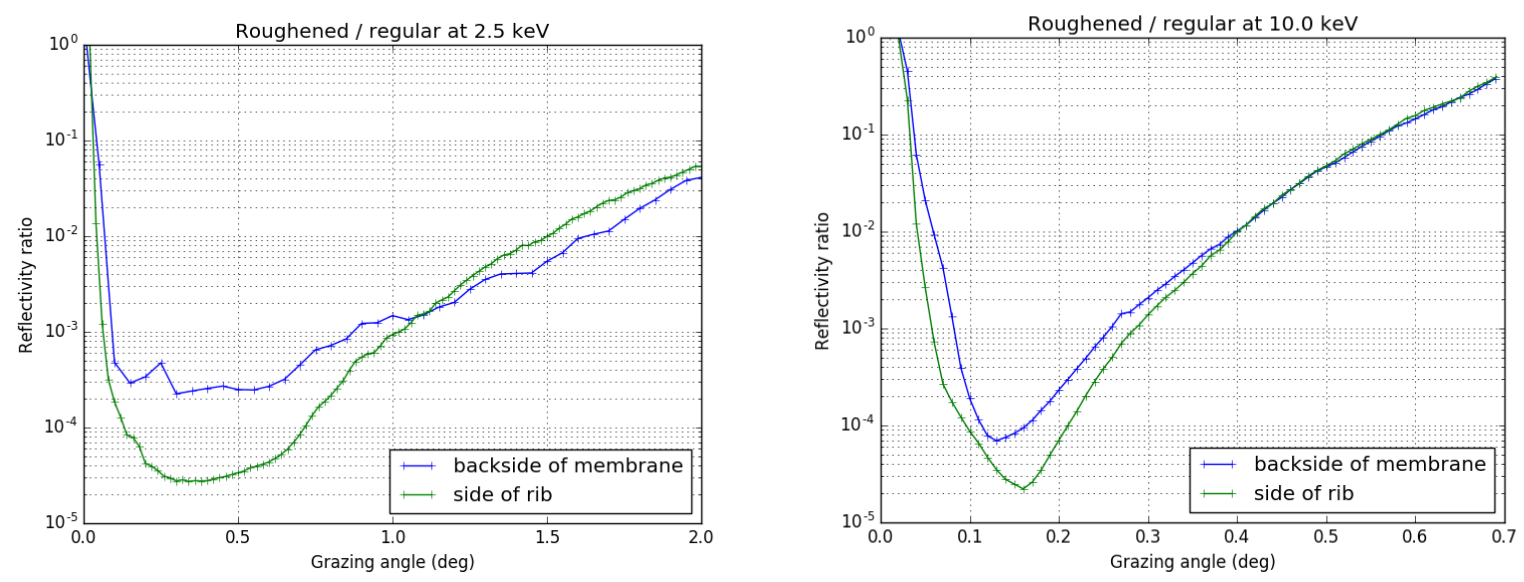

Figure 4 - Reflectometry comparing the ratio the reflectivity of a regular to a roughened plate as a function of grazing incidence angle, at two different energies $(2.5 \mathrm{keV}$ left, $10 \mathrm{keV}$ right). The data was recorded at the FCM beamline of PTB at BESSY II. The reflectivity of both the ribbed side and the backside of the silicon mirror can be reduced by several orders of magnitude as a result of the blackening process.

This means, if Athena requires it, we would have a technique to adjust the reflectivity of the backside of plates and if necessary, can include it in time for the MAR in the mass production process. Other areas of plate development address thinning the membrane to gain effective area and widening the rib spacing to increase the field of view.

\section{PLATE COATING}

\subsection{Preparation of coated plate mass production}

In April 2018 a large drum coating machine using magnetron sputtering to deposit thin film coatings on the mirror plates has been delivered and installed at cosine, next to the stacking robots. This marks an important step to demonstrate the mass coating capability at the MAR, as so far all representative coated stacks have been produced with smaller scale coating systems. The industrial system has been custom designed and holds up to three magnetrons. It also has a plasma cleaning system and can coat up to 150 plates in one fully automated run, with a capacity of about 100.000 plates per year.

The plates are supplied with lithographic masking by the plate suppliers and are loaded into frames that are hung into a carrousel inside the coating machine, through a large door from the cleanrooms (see Figure 5). The machine itself is located in a grey-room and can be serviced and cleaned from there. The system is then evacuated and automatically performs the coating run. After several hours, depending on the coating recipe, the machine is vented and the coated mirror plates undergo a lift-off process before they are being stacked. We are currently in the process of performing onsite acceptance tests and will then continue to commission the system until the end of the year. We will then use the coating system to produce the coated stacks required for the QM.

\subsection{Coating process development}

The coating process itself is currently being developed by DTU Space [20-22] and is then applied and tested on the industrial coating machine described above. First results of single, bi- and multi-layers of Ir and $\mathrm{B}_{4} \mathrm{C}$ coated on silicon using the industrial coating machine show very good results, demonstrating that the research grade quality can be matched with the mass production systems being prepared for Athena. In order to follow the developments of DTU on the Athena baseline coating design, we are in the process of also procuring a SiC target. In 2018 we will complete the commissioning of the coating machine, by DTU carrying out studies on the uniformity of the coating, the plasma cleaning, collimation effects, film structure and preliminary composition studies. In parallel to the coating process development we also work with the plate suppliers and DTU on the improvement of the lithographic masking process. The plates only need to be coated in between the ribs, to leave the bonding areas to be silicon oxide. For small volume 
production the currently used process is sufficient, however for larger volumes dedicated lithography equipment and suitably sized lift-off baths will have to be developed, commissioned and tested.
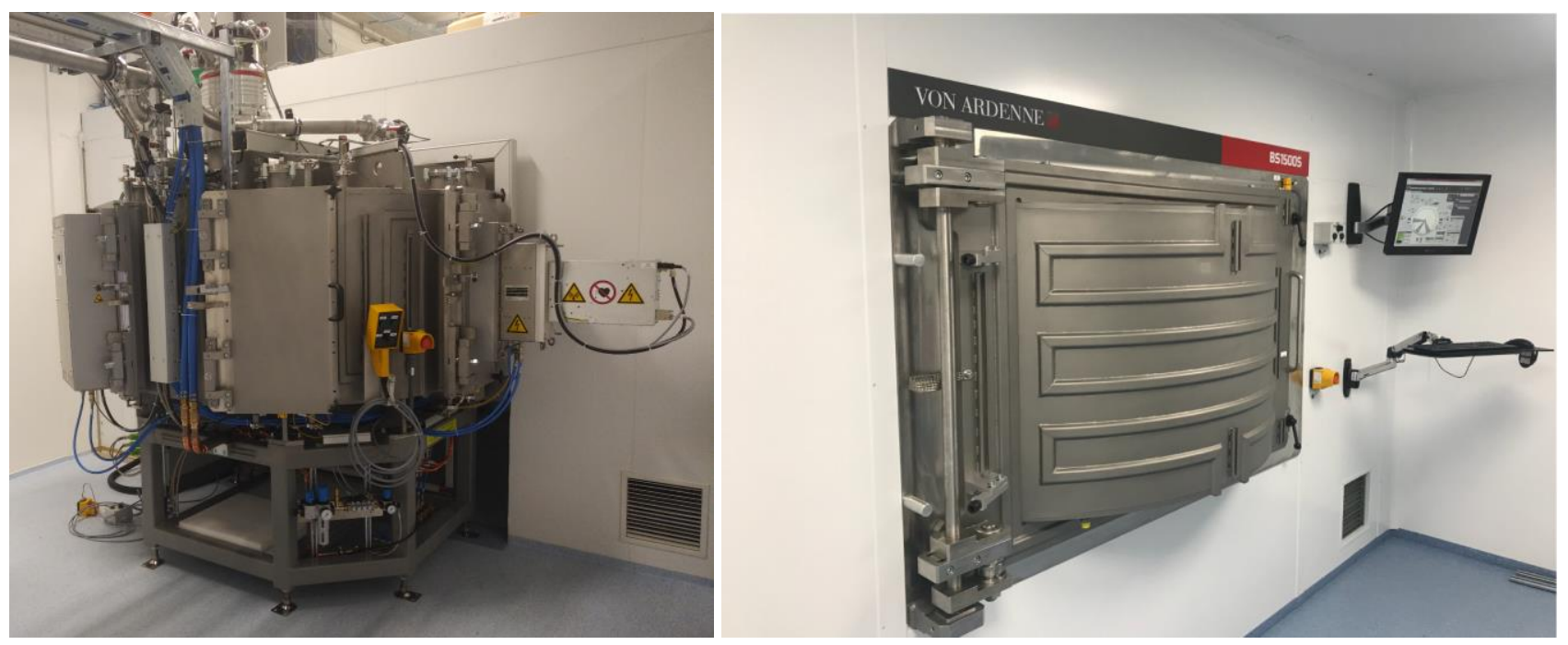

Figure 5 - Photographs of the newly installed drum coating machine for mass production of coated SPO plates. The left image shows the backside of the machine inside a grey room for easy access and service. The machine has a plasma cleaner, two magnetrons and space for a third one. The right image shows the front side of the vacuum chamber as seen from inside the cleanroom of cosine. The large door can be opened to load and unload carriers holding up to 150 SPO plates. The system is then closed, evacuated and the carousel moves the plates past the magnetrons to sputter single or multi-layers. The system is fully automated and the recipe can be set via the computer system shown in the right image.

\section{STACKING OF PLATES INTO MIRROR MODULES}

\subsection{Preparation of stack mass production}

In 2017 we performed a first production run for second generation $f=12 \mathrm{~m}$ middle radii mirror modules, to meet the requirement to supply 4 mirror modules [23] for the two parallel Assembly, Integration and Verification (AIT) activities $[24,25]$ of ESA, another part of ESA's TDP plan, to develop methods to co-align and integrate mirror modules into the Athena optical bench. Such a production run allows testing all parts of the stacking chain, from plate inspection, cleaning to stacking on two different types of mandrels, using several stacking robot operators working in shifts, to x-ray testing them at XPBF 1 (X-ray pencil beam facility) [26, 27] or the new XPBF 2.0 (X-ray parallel beam facility) [28, 29], both operated by PTB at the BESSY II synchrotron radiation facility. As preparation for the production run we had in the first half of 2017 upgraded the stacking facilities, water supplies, control equipment and trained additional stacking robot operators. We also reduced the stacking times from 45 minutes per plate to less than 20 minutes. Furthermore we upgraded the data analysis pipelines in order to be quicker in the characterization of stacks and mirror modules. We successfully produced a total of 18 stacks with a height of 35 plates, consuming more than 600 plates within a few months. Of these, 16 were integrated into 4 mirror modules (see Figure 6), within a period of 6 weeks.

From the production run we also learned valuable lessons which are being addressed this year to prepare for the MAR in 2021:

- The process to align and assemble 4 stacks into the mirror modules using manually operated jigs was rather time consuming. This has led to the development of a motorized integration jig, using 3 hexapods integrated in a system that can be moved in and out of the vacuum chamber of XPBF 2.0 (see Figure 7). The system is now being used to make in 2018 the first truly confocal mirror module where both stack pairs are made using stacks from two different radii of $709 \mathrm{~mm}$ and $737 \mathrm{~mm}$. 
- The stacks produced showed a variability in the alignment of their mechanical axis, defined by the ribs, with respect to the optical axis defined by the mandrel, of up to 10 arc minutes, resulting in vignetting of the pores in double reflection. We have addressed this by developing and commissioning a dedicated metrology system installed on the stacking robots that measures the alignment of the ribs of the baseplate of a stack to the mandrel optical axis to an accuracy of about 1 arc minute.
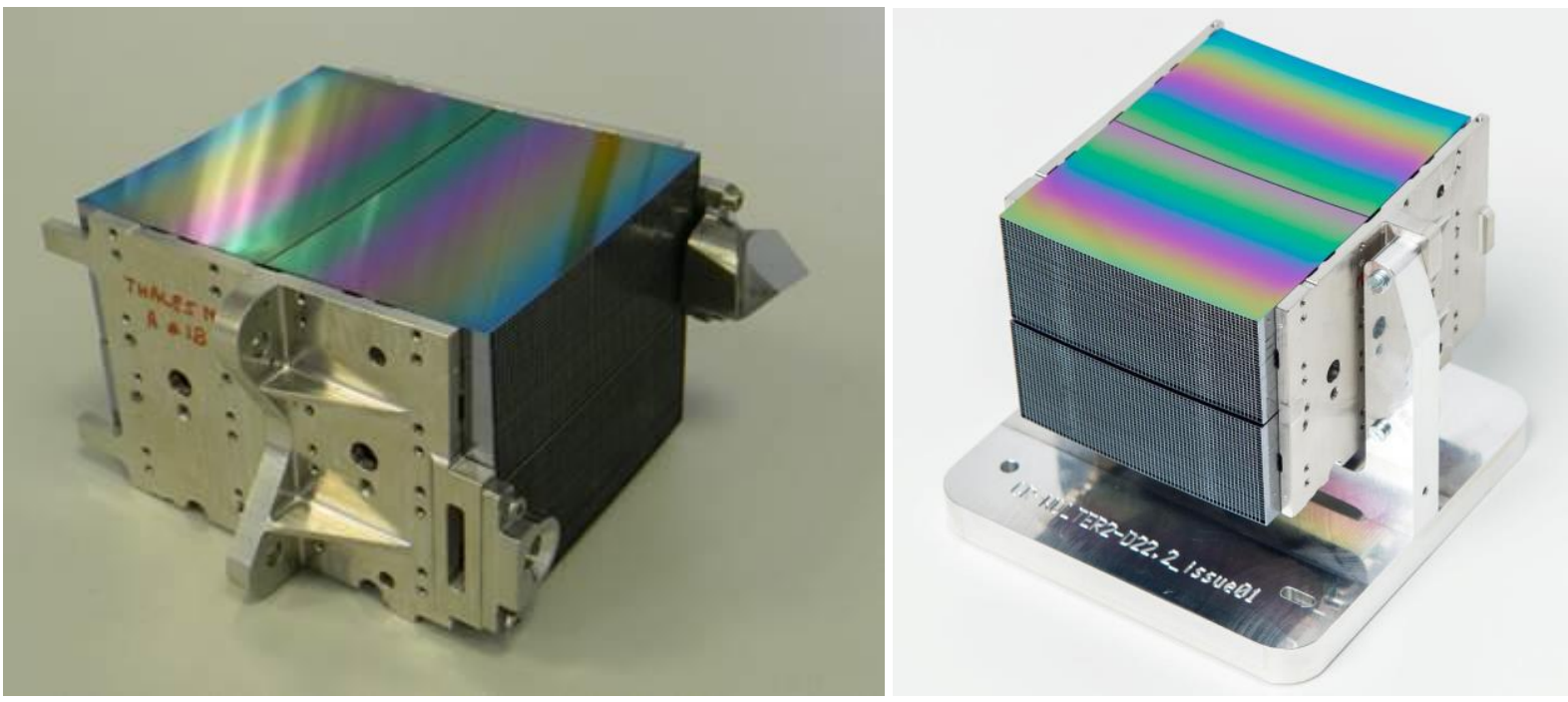

Figure 6 - Photos of two of the four mirror modules, differentiated by their bracket type, produced during the production run in 2017. The left image shows a mirror module prepared for the AIT activities led by TAS Switzerland, the right photo shows a Media Lario module. Each mirror module consists of 2 pairs, called an XOU, of a primary and a secondary mirror stack.

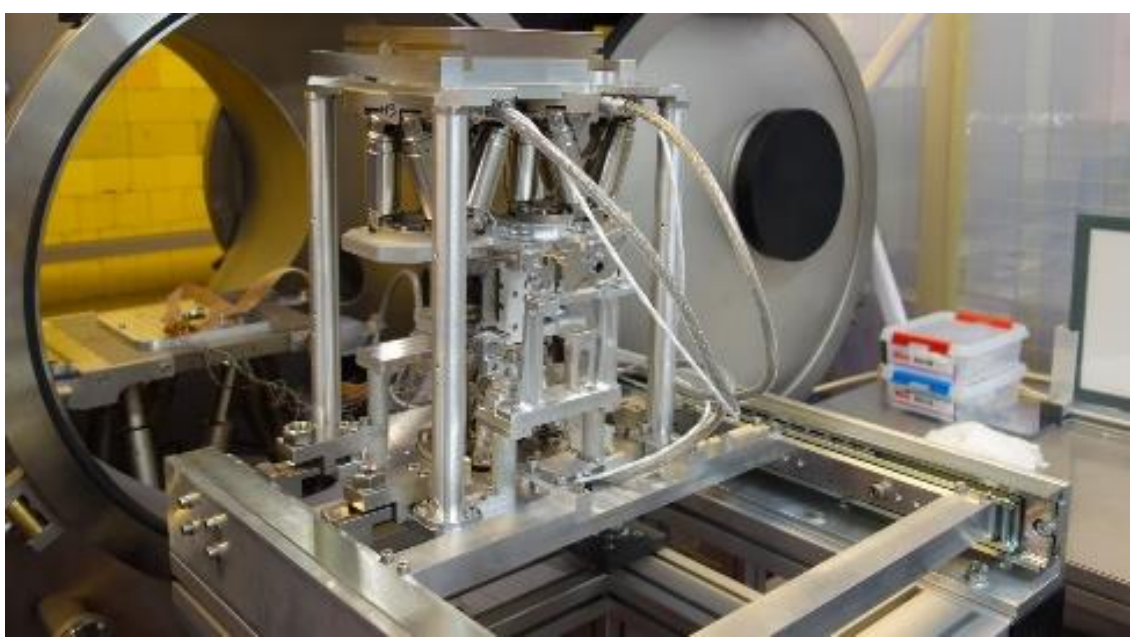

Figure 7 - Motorised $2^{\text {nd }}$ generation mirror module alignment and assembly jig, outside of the vacuum chamber of XPBF 2.0 of PTB at BESSY II. It has three hexapods to co-align in vacuum and under $x$-ray illumination 3 stacks to the fourth one. After alignment, the chamber is vented and the jig can be moved outside of the chamber to inject glue to fixate the brackets to the stacks. 


\subsection{Stacking process development}

We had produced a very first set of $f=12 \mathrm{~m}$ middle radii test stacks late 2016 to test the new production equipment. The x-ray test resulted in a $\sim 40$ " HEW PSF (see XOU-0038 in Figure 8), as is to be expected when commissioning new production systems where all relevant parts such as plates, mandrels and dies had to be adjusted to accommodate the focal length change. After we had further upgraded the production facilities as described above, we could use several months in 2017 to improve the stacking process parameters, resulting in a performance improvement of a factor of $\sim 2$ (XOU-0044 in Figure 8), catching up with the $f=20 \mathrm{~m}$ angular resolution (XOU-0035). An in-depth review of the stacking results carried out early 2018 has shown the plate pre-forming equipment to cause figure deviations at the edges of the plates and we are now in the process of adjusting the tooling to improve the performance further, resulting later this year in $3^{\text {rd }}$ generation optics. This process improvement is part of ESA's TDP, where a dedicated activity focusses on improving the angular resolution in several iterations.

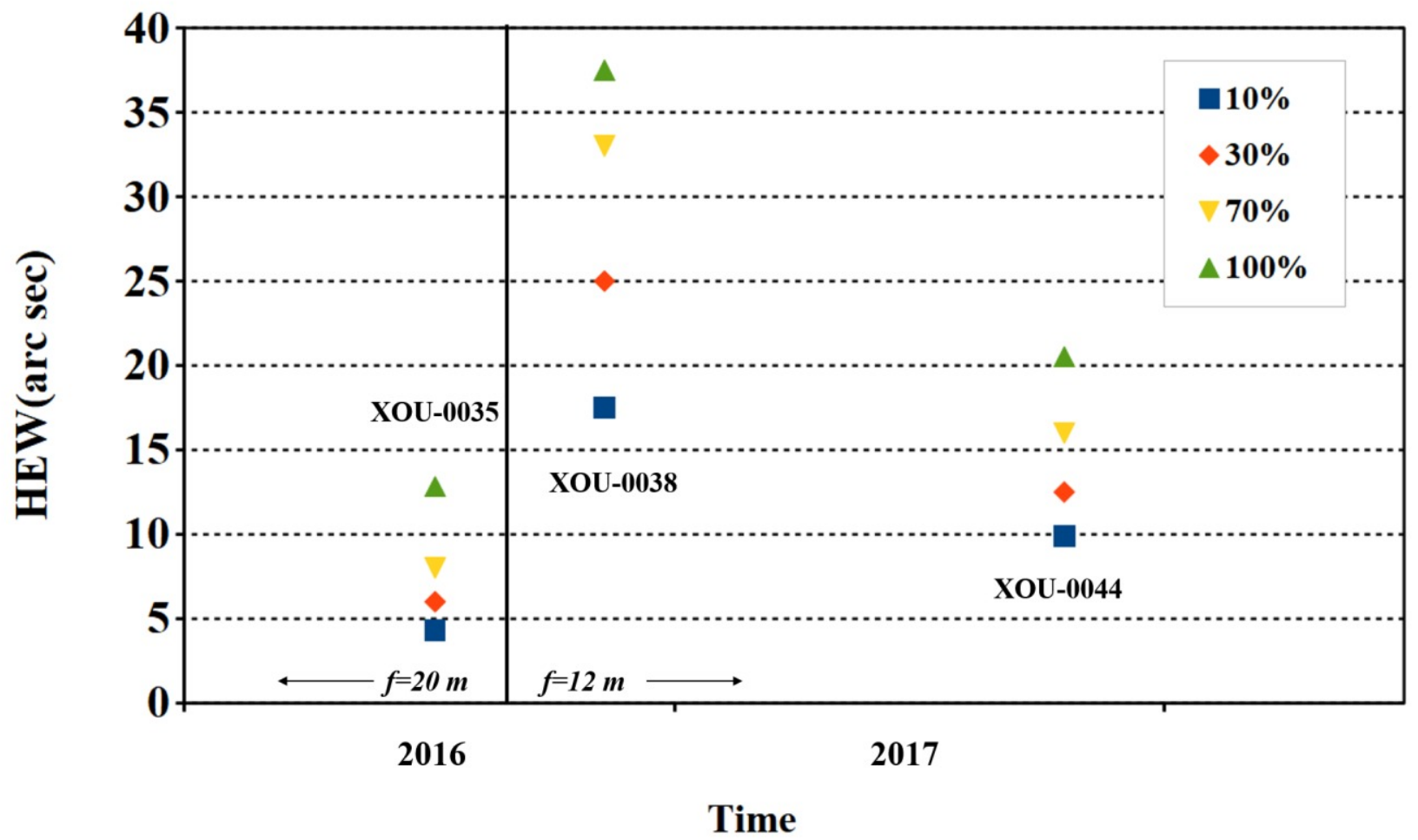

Figure 8 - The half energy width (HEW) of 20 plates of several XOUs as measured in double reflection at XPBF 1 in the PTB laboratory at BESSY II. XOU-0035 is the last $f=20 \mathrm{~m}$ optics produced in July 2016, measured at $2.8 \mathrm{keV}$. XOU-0038 is the first generation $f=12 \mathrm{~m}$ optics, XOU-0044 is of the $2^{\text {nd }}$ generation $f=12 \mathrm{~m}$ optics, all measured at $1.0 \mathrm{keV}$. We show data of $10 \%, 30 \%, 70 \%$ and $100 \%$ of the width of the XOU area illuminated by the $x$-ray beam. This year we are further improving on the stacking process to produce a $3^{\text {rd }}$ generation XOU.

The outer radii development runs in parallel to the middle radii. We have recently reached an important milestone of producing the very first stacks with a nominal height of 35 plates (see Figure 9). The development now focuses on improving the cleanliness of the plates and on stacking iridium coated plates, required to measure the stacks in $\mathrm{x}$-rays at an energy of $1 \mathrm{keV}$, as the grazing incidence angle of $1.78 \mathrm{deg}$ at $r=1500 \mathrm{~mm}$ would result in a very low silicon reflectivity. In 2018 we are going to produce several 35 plate stacks to be tested in double reflection and to be finally integrated into a first outer radius mirror module.

The inner radii development had demonstrated a first 35 plate stack late 2016 and has essentially been on hold since then, to give priority to the other activities discussed in this paper. We are in the process of setting up an additional 
stacking robot and will start the production of inner radii stacks later in 2018, such that all three representative types of mirror modules can be produced in 2019 in preparation for the Qualification Model demonstration required for the MAR in 2021.
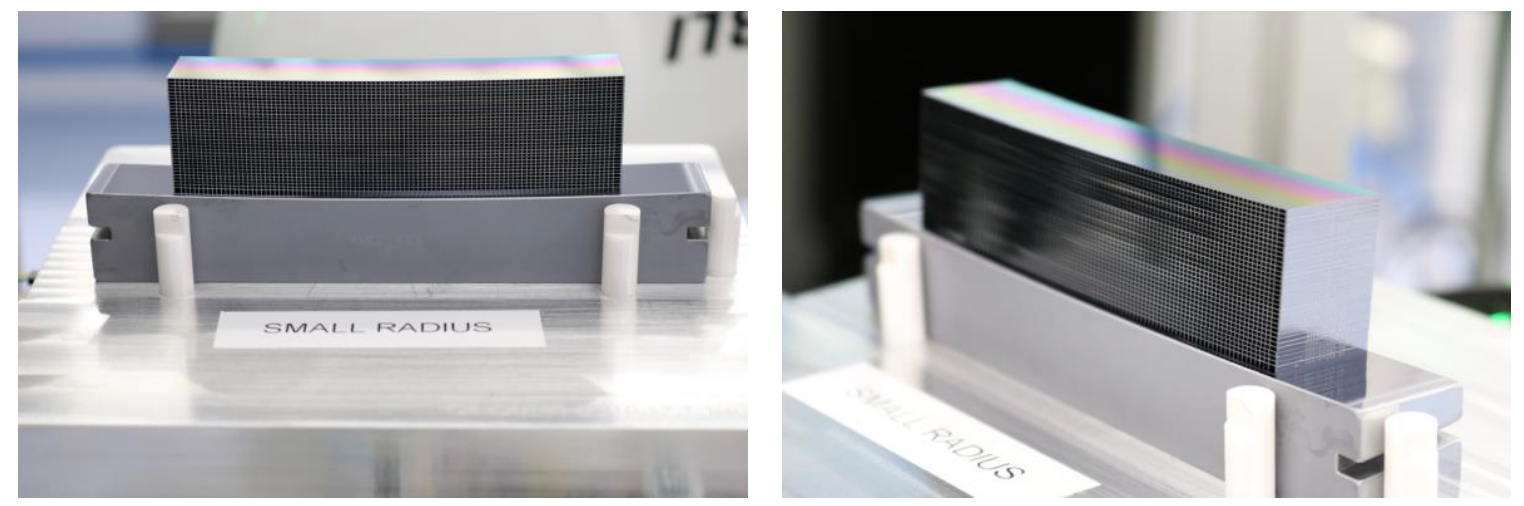

Figure 9 - Photographs of the very first 35 plate outer radius stack, produced in May 2018.

\section{ENVIRONMENTAL TESTING}

The development of SPO in ESA's TDP also includes an activity that focuses on environmental testing of the stacks and mirror modules. The goal is to continuously explore the failure modes of the optics, in parallel to the ongoing process development. The shock requirements for Athena are being reviewed by ESA as part of two parallel system studies and in view of the information that is being released about the levels to be expected by the Ariane 6 launcher. We currently work with a requirement that SPO mirror modules have to pass a $350 \mathrm{~g}$ shock and have developed a dedicated shock table to carry out regular tests. As a first part of the activity we carry out regular shock tests [30] on middle radius stacks, to compare the failure modes and to quantify the influence of process changes to the bond strength. We have found that stacks pass typically between $600 \mathrm{~g}$ to $1000 \mathrm{~g}$ shocks and if we apply a thermal annealing they pass levels of 930 to $1340 \mathrm{~g}$, with the only failure mode observed so far being a debonding of a mirror plate. We will now further develop the annealing process to maximize the strength of the optics. As a next step we will start to shock outer and inner radii stacks and begin testing middle radius mirror modules.

\section{CONCLUSION}

The Athena optics development is moving ahead, following the technology development plan of ESA to mature the technology to TRL 6 for the Mission Adoption Review (MAR) expected in 2021. We have shown in this paper that all areas of the SPO mirror modules development are making progress, both on the mass production and on the quality aspects. This includes the timely preparation of production capabilities to demonstrate the schedule and cost compliance for mass producing the Athena optics.

All three mirror plate types required for the MAR can be produced in larger volume and we are also qualifying a second plate supplier. The coating process is being scaled up from smaller research production to mass production and the installation of a large coating machine has been completed. First production runs of middle mirror modules were successfully completed in 2017 and the stack quality has improved by a factor of 2 compared to the first generation $f=12 \mathrm{~m}$ optics. Outer radii stacks have reached their nominal height of 35 plates. Shock testing of stacks produces consistent results. The development can therefore focus the following year mainly on improving the angular resolution further to meet the requirements, which is being done in a systematic manner mainly by improving the pre-forming tools used in the stacking robots. 


\section{REFERENCES}

[1] Jansen, F. et al., "XMM-Newton observatory", Astron. Astrophys. 365, L1-L6 (2001)

[2] O'Dell, S.L. and M. C. Weisskopf ,"Advanced X-ray astrophysics facility (AXAF): Calibration overview”, Proc. SPIE 3444 (1998) and references therein

[3] K. Nandra, "Athena: Exploring the Hot and Energetic Universe", Proc. American Astronomical Society HEAD meeting (\#14), (2014).

[4] Ayre, M., et al, "ATHENA - System design and implementation for a next generation x-ray telescope", Proc. of SPIE Vol. 9601, 96010L (2015)

[5] Ayre, M., et al, "ATHENA: system studies and optics accommodation", Proc. SPIE 9905, 990526 (2016)

[6] Ayre, M., et al, "ATHENA - System design and implementation for a next generation x-ray telescope", Proc. of SPIE Vol. 10397, 103970X-2 (2017)

[7] Meidinger, N. et al., "The Wide Field Imager instrument for Athena", Proc. SPIE 10397-12 (2017)

[8] Barret, D. et al., "The Athena X-ray Integral Field Unit”, Proc. SPIE 9905 (2016)

[9] Wolter, H., "Spiegelsysteme streifenden Einfalls als abbildende Optiken für Röntgenstrahlen", Annalen der Physik 445, 94-114 (1952).

[10] R. Willingale, G. Pareschi, F. Christensen, J-W. den Herder, "The Optical Design of the Athena+ Mirror", An ATHENA+ supporting paper, <http://www.the-athena-X-ray-observatory.eu/

[11] Wille, E. et al., "Silicon pore optics manufacturing plan and schedule for ATHENA", these proceedings

[12] Beijersbergen, M. et al., "Silicon pore optics: novel lightweight high-resolution X-ray optics developed for XEUS", Proc. SPIE 5488, 868-874 (2004).

[13] Bavdaz, M. et al., "Progress at ESA on high-energy optics technologies", Proc. SPIE 5168, 136-147 (2004).

[14] Smith, R. K., el al, “Arcus: The X-ray Grating Spectrometer Explorer”, Proc. SPIE 10397 (2017).

[15] Collon, M., et al, "Silicon pore optics for the ATHENA telescope", Proc. SPIE 9905 (2016).

[16] Bavdaz, M., et al, "The ATHENA Telescope and Optics Status", Proc. SPIE 10399 (2017).

[17] Bavdaz, M., et al., "Development of the ATHENA mirror", same proceedings

[18] Olde Riekerink, M. B. et al. "Production of silicon mirror plates", Proc. SPIE 7437, 74370U (2009)

[19] Krumrey, M. and G. Ulm, "High accuracy detector calibration at the PTB four-crystal monochromator beamline", Nucl. Instr. and Meth. A 467-468, 1175 - 1178 (2001).

[20] Della Monica Ferreira, D., et al, "Design, development, and performance of X-ray mirror coatings for the ATHENA mission", Proc. SPIE 10399 (2017).

[21] Massahi, S., et al, "Industrialization of the mirror plate coatings for the Athena mission", Proc. SPIE 10399 (2017).

[22] Della Monica Ferreira, D., et al., "Performance and Stability of Mirror Coatings for the ATHENA Mission", same proceedings

[23] Barrière, N. M., et al, "Silicon pore optics mirror module assembly process", Proc. SPIE 10399 (2017).

[24] Valsecchi, G. et al, "Optical integration of the SPO modules in the ATHENA telescope", Proc SPIE 10399 (2017).

[25] Vernani, D., et al, "Integration of the Athena mirror modules: development of indirect and X-ray direct AIT methods", Proc SPIE 10399 (2017).

[26] Krumrey, M., et al, "X-ray pencil beam facility for optics characterization", Proc. SPIE 7732, 773240 (2010).

[27] Vacanti, G, et al., "Silicon pore optics for astrophysical missions", Proc. SPIE 7732, 773240 (2010).

[28] Krumrey, M., et al, "New X-ray parallel beam facility XPBF 2.0 for the characterization of silicon pore optics", Proc. SPIE 9905 (2016).

[29] Vacanti, G., et al, "New ray-tracing capabilities for the development of silicon pore optics", Proc. SPIE 9603 (2015).

[30] Landgraf, B., et al, "Environmental testing of the Athena mirror modules", Proc. SPIE 10399 (2017). 